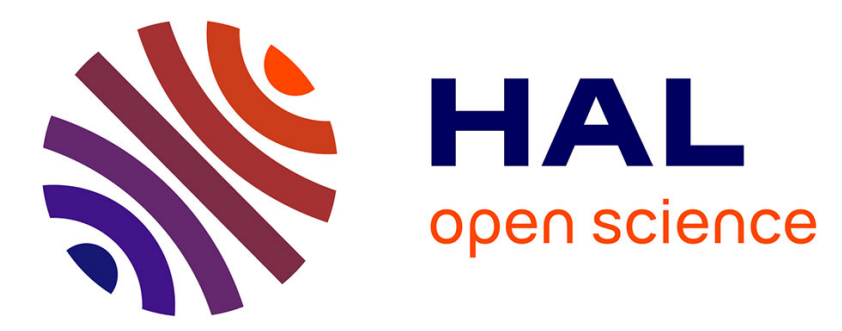

\title{
Stability analysis of a Vlasov-Wave system describing particles interacting with their environment
}

\author{
Stephan de Bièvre, Thierry Goudon, Arthur Vavasseur
}

\section{To cite this version:}

Stephan de Bièvre, Thierry Goudon, Arthur Vavasseur. Stability analysis of a Vlasov-Wave system describing particles interacting with their environment. Journal of Differential Equations, 2018, 264 (12), pp.7069-7093. hal-01581676

\section{HAL Id: hal-01581676 \\ https://hal.inria.fr/hal-01581676}

Submitted on 5 Sep 2017

HAL is a multi-disciplinary open access archive for the deposit and dissemination of scientific research documents, whether they are published or not. The documents may come from teaching and research institutions in France or abroad, or from public or private research centers.
L'archive ouverte pluridisciplinaire HAL, est destinée au dépôt et à la diffusion de documents scientifiques de niveau recherche, publiés ou non, émanant des établissements d'enseignement et de recherche français ou étrangers, des laboratoires publics ou privés. 


\title{
Stability analysis of a Vlasov-Wave system describing particles interacting with their environment
}

\author{
Stephan De Bièvre*1, Thierry Goudon ${ }^{\dagger 2}$, and Arthur Vavasseur ${ }^{\ddagger 2}$ \\ ${ }^{1}$ Univ. Lille, CNRS, UMR 8524 - Laboratoire Paul Painlevé, F-59000 Lille, France ${ }^{\S}$ \\ \& Equipe-Projet MEPHYSTO, Centre de Recherche INRIA Lille Nord Europe, \\ Parc Scientifique de la Haute Borne, 40, avenue Halley B.P. 70478 \\ F-59658 Villeneuve d'Ascq cedex, France. \\ ${ }^{2}$ Université Côte d'Azur, Inria, CNRS, LJAD, \\ Parc Valrose, F-06108 Nice, France
}

\begin{abstract}
We study a kinetic equation of the Vlasov-Wave type, which arises in the description of the behaviour of a large number of particles interacting weakly with an environment, composed of an infinite collection of local vibrational degrees of freedom, modeled by wave equations. We use variational techniques to establish the existence of large families of stationary states for this system, and analyze their stability.
\end{abstract}

Keywords. Vlasov-like equations. Interacting particles. Dynamical stability. Inelastic Lorentz gas.

Math. Subject Classification. 82C70, 70F45, 37K05, 74A25.

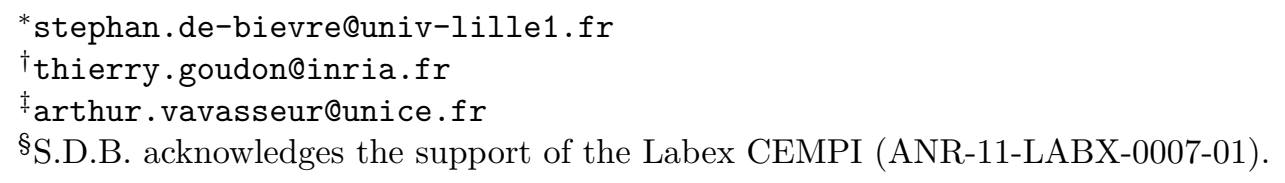




\section{Introduction}

This paper is concerned with the following PDE system

$$
\begin{array}{r}
\partial_{t} f+v \cdot \nabla_{x} f-\nabla_{x}(V+\Phi) \cdot \nabla_{v} f=0, \quad t \geq 0, x \in \mathbb{R}^{d}, v \in \mathbb{R}^{d}, \\
\left(\partial_{t t}^{2} \Psi-c^{2} \Delta_{z} \Psi\right)(t, x, z)=-\sigma_{2}(z) \int_{\mathbb{R}^{d}} \sigma_{1}(x-y) \rho(t, y) \mathrm{d} y, t \geq 0, x \in \mathbb{R}^{d}, z \in \mathbb{R}^{n}, \\
\rho(t, x)=\int_{\mathbb{R}^{d}} f(t, x, v) \mathrm{d} v, \\
\Phi(t, x)=\iint_{\mathbb{R}^{d} \times \mathbb{R}^{n}} \Psi(t, y, z) \sigma_{1}(x-y) \sigma_{2}(z) \mathrm{d} z \mathrm{~d} y, \quad t \geq 0, x \in \mathbb{R}^{d} .
\end{array}
$$

This system was introduced in $[8,25]$, where also the existence of solutions was established and some asymptotic issues treated. In particular, a certain asymptotic regime brings out a quite unexpected connection between this system and the gravitational Vlasov-Poisson system. In this work, we wish to address a first aspect of the long-time behaviour of the system by investigating the existence of stationary solutions and their stability.

We recall the system (1)-(4) describes the interaction between a large set of particles and their environment. The particles are described by their distribution function in phase space: $f(t, x, v)$, where $t \geq 0, x \in \mathbb{R}^{d}, v \in \mathbb{R}^{d}$ are the time, space and velocity variables, respectively. More precisely, we interpret this quantity so that, given $\mathscr{O} \times \mathscr{V} \subset$ $\mathbb{R}^{d} \times \mathbb{R}^{d}, \iint_{\mathscr{O} \times \mathscr{V}} f(t, x, v) \mathrm{d} v \mathrm{~d} x$ gives the mass of particles having at time $t \geq 0$ their position in $\mathscr{O}$ and their velocity in $\mathscr{V}$. The environment is seen as a vibrating medium, the state of which is described by the function $\Psi$. Note that the vibrations take place in the direction $z \in \mathbb{R}^{n}$, which is transverse to the particles' motion; they are characterized by the wave speed $c>0$. The coupling between the particles and the environment is embodied in the form functions $\sigma_{1}, \sigma_{2}$, which are both non negative, smooth and radially symmetric functions. Finally, the particles are also subjected to a confining potential $V$. We shall give details later on the technical assumptions and start by discussing the modelling issues.

The system (1)-(4) is a kinetic version of a model introduced by L. Bruneau and S. De Bièvre [5] for a single classical particle interacting with its environment. It is useful to keep in mind the picture proposed in [5] of a particle moving trough an infinite set of $n$-dimensional membranes, one for each $x \in \mathbb{R}^{d}$. Denoting by $t \mapsto q(t)$ the position of the particle, the dynamic of such a particle is described by the following set of differential equations

$$
\left\{\begin{array}{l}
\ddot{q}(t)=-\nabla V(q(t))-\iint_{\mathbb{R}^{d} \times \mathbb{R}^{n}} \sigma_{1}(q(t)-y) \sigma_{2}(z) \nabla_{x} \Psi(t, y, z) \mathrm{d} y \mathrm{~d} z, \\
\partial_{t t}^{2} \Psi(t, x, z)-c^{2} \Delta_{z} \Psi(t, x, z)=-\sigma_{2}(z) \sigma_{1}(x-q(t)), \quad x \in \mathbb{R}^{d}, z \in \mathbb{R}^{n} .
\end{array}\right.
$$

For the system (5), due to the energy exchanges between the particles and the environment, and the evacuation of energy in the membranes, the coupling eventually 
behaves like a friction force on the particle so that the particle asymptotically stops at the minimum of the confining potential $V$. Further situations are dealt with in [5], and we also refer the reader to $[1,9,10,11,21,24]$ for thorough numerical experiments and analytical studies on (5) and related models. The system (5) can be generalized by considering a set of $N$ particles, and the mean field regime $N \rightarrow \infty$ leads to (1)-(4), see $[17,25]$.

In [2], hypocoercivity techniques are adapted from [13] to investigate the large time time behavior of the solutions, when the kinetic equation (1) additionaly contains the Fokker-Planck operator $\nabla_{v} \cdot\left(v f+\nabla_{v} f\right)$. This operator induces some dissipative effects in the model which simplifies the asymptotic analysis. However, the stationary solution

$$
\mathscr{M}_{\text {eq }}(x, v)=Z_{\text {eq }} \exp \left(-\frac{v^{2}}{2}-V(x)-\Phi_{\text {eq }}(x)\right)
$$

exhibited in $[2,25]$ defines also a stationary solution of (1)-(4). It is then natural to investigate the stability of this solution, a task we turn to in this paper. In (6), $\Phi_{\text {eq }}$ is implicitely defined by the non-linear equation

$$
\Phi_{\mathrm{eq}}(x)=-\frac{\kappa}{c^{2}} \sigma_{1} \star \sigma_{1} \star \int_{\mathbb{R}^{d}} \mathscr{M}_{\mathrm{eq}}(x, v) \mathrm{d} v, \quad \kappa=\int_{\mathbb{R}^{n}} \frac{\left|\widehat{\sigma}_{2}(\xi)\right|^{2}}{\xi^{2}} \mathrm{~d} \xi>0 .
$$

Note that the definition of $\kappa$ requires $n \geq 3$. It can be equivalently expressed as

$$
\kappa=\int_{\mathbb{R}^{n}}|\nabla \Gamma(z)|^{2} \mathrm{~d} z>0
$$

with $\Gamma$ the solution of

$$
\Delta \Gamma(z)=\sigma_{2}(z), \quad z \in \mathbb{R}^{n} .
$$

Equation (6) defines a family of stationary solutions of (1)-(4), parametrized here by $Z_{\text {eq }} \geq 0$. Since (1) conserves the total mass, we address the question of the stability of the stationary solution which has the same mass as the initial data, namely $Z_{\text {eq }}$ is the normalizing constant such that

$$
\iint_{\mathbb{R}^{d} \times \mathbb{R}^{d}} \mathscr{M}_{\mathrm{eq}}(x, v) \mathrm{d} v \mathrm{~d} x=\iint_{\mathbb{R}^{d} \times \mathbb{R}^{d}} f(0, x, v) \mathrm{d} v \mathrm{~d} x=\iint_{\mathbb{R}^{d} \times \mathbb{R}^{d}} f(t, x, v) \mathrm{d} v \mathrm{~d} x .
$$

In $[2,25]$ this solution is shown to be linearly stable for the collisionless system (1)-(4). We wish to investigate further the issue of the existence of these and other stationary solutions and their non-linear stability. We point out that the large time behavior for the kinetic model (1)-(4), or even for the $N$-particles version of (5), differs considerably from the situation investigated in [5] for a single particle, precisely because of the intricate interactions between all the particles when they are close to each other. When dealing with a single particle, with $V$ a confining potential as considered here, the asymptotic behavior is quite simple. Assuming for simplicity the potential has a unique critical point, which is its absolute minimum, it was shown the particle comes (exponentially fast) to rest at this minimum, see [5, Theorem 4]. This is - intuitively at least - a consequence of the fact that, as long as the particle moves, it will loose energy to the membranes, so that it has to come to a full stop. Which it can only do 
where no external force is applied by the external potential. With several particles, the analysis is not that simple since when two particles are close enough to each other, they activate the membranes in a common neighborhood, which could lead to more complicated energy balances between the particle and the membranes. So, while a stationary solution with all particles at the minimum of the potential still exists, it is thus not clear if other stationary solutions may or may not exist. For the kinetic model, which corresponds to the statistical description of many particles in a mean field regime when the coupling between the particles is weak, we shall see that the model admits infinitely many stationary solutions with different shape.

In this paper we thus go back to the stationary solutions of the Vlasov-Wave system (1)-(4). Using the Hamiltonian structure at the basis of the discussion in [5], and the conservation of the Casimir-functionals of the Vlasov equation (1), we develop a variational approach, strongly inspired by the analysis of the gravitational VlasovPoisson system [14, 18, 19, 23, 26]; see also [3, 6] for the electrostatic Vlasov-Poisson system. In Section 2 we discuss the existence of stationary solutions in connection to the minimization of suitable energy functionals. Section 3 investigates the dynamical stability of these stationary solutions. Whether analogous stationary states also exist in the original $N$-particle model is a difficult open question that we do not address here and that is related to the question of the time-scale on which the Vlasov-Wave equation correctly describes the $N$-particle model.

\section{Stationary solutions}

A first crucial observation is that (1)-(4) conserves an energy functional that encodes the energy exchanges between the particles and their environment:

$$
\begin{aligned}
\mathscr{E}(f, \Psi, \pi)=\iint_{\mathbb{R}^{d} \times \mathbb{R}^{d}} & \left(\frac{v^{2}}{2}+V(x)\right) f \mathrm{~d} v \mathrm{~d} x+\iint_{\mathbb{R}^{d} \times \mathbb{R}^{d}} \Phi[\Psi] f \mathrm{~d} v \mathrm{~d} x \\
+ & \frac{1}{2} \iint_{\mathbb{R}^{d} \times \mathbb{R}^{n}}\left(|\pi|^{2}+c^{2}\left|\nabla_{z} \Psi\right|^{2}\right) \mathrm{d} y \mathrm{~d} x .
\end{aligned}
$$

Here we have used the following notation: for a function $\Psi: \mathbb{R}^{d} \times \mathbb{R}^{n} \rightarrow \mathbb{R}$,

$$
\Phi[\Psi](x)=\int_{\mathbb{R}^{d}} \sigma_{1}(x-y)\left(\int_{\mathbb{R}^{n}} \sigma_{2}(z) \Psi(y, z) \mathrm{d} z\right) \mathrm{d} y .
$$

Then, we have energy conservation in the sense that the solutions of (1)-(4) satisfy

$$
\mathscr{E}\left(f, \Psi, \partial_{t} \Psi\right)(t)=\mathscr{E}\left(f, \Psi, \partial_{t} \Psi\right)(0) .
$$

Of course, the particles' total mass is also conserved

$$
\iint_{\mathbb{R}^{d} \times \mathbb{R}^{d}} f(t, x, v) \mathrm{d} v \mathrm{~d} x=\iint_{\mathbb{R}^{d} \times \mathbb{R}^{d}} f(0, x, v) \mathrm{d} v \mathrm{~d} x .
$$

Finally, since the field $(x, v) \mapsto\left(v,-\nabla_{x} V(x)-\nabla_{x} \Phi(t, x)\right)$ is divergence-free, equation (1) also conserves any non-linear functional (the "Casimir-functionals")

$$
\iint_{\mathbb{R}^{d} \times \mathbb{R}^{d}} h(f(t, x, v)) \mathrm{d} v \mathrm{~d} x=\iint_{\mathbb{R}^{d} \times \mathbb{R}^{d}} h(f(0, x, v)) \mathrm{d} v \mathrm{~d} x
$$


for a given function $h:[0, \infty) \rightarrow \mathbb{R}$.

This suggests to search for stationary solutions of (1)-(4) as minimizers of a Lyapounov functional using (8), (9) and (10). The natural energy (7) does not have enough structure to serve as a Lyapounov functional for the evolution problem. The idea is thus to combine (7) and (10) and to study the critical points of this Casimirenergy functional, under a mass constraint. It turns out that the critical points are also stationary solutions of (1)-(4). Moreover, the minimization property defines the framework that allows us to investigate the stability issue. We refer for instance the reader to $[3,6,12,18,26]$ for similar reasonings, see also the overview [23]. We proceed as follows. Let $h:[0, \infty) \rightarrow \mathbb{R}$ be a strictly convex function. We set

$$
\mathscr{J}(f, \Psi, \pi)=\iint_{\mathbb{R}^{d} \times \mathbb{R}^{d}} h(f) \mathrm{d} v \mathrm{~d} x+\mathscr{E}(f, \Psi, \pi) .
$$

Let us denote by $m$ the mass of the initial particle distribution function

$$
m=\iint_{\mathbb{R}^{d} \times \mathbb{R}^{d}} f(0, x, v) \mathrm{d} v \mathrm{~d} x .
$$

We wish to define $\left(\mathscr{M}_{\text {eq }}, \Psi_{\text {eq }}, \pi_{\text {eq }}\right)$ as the minimizer of $\mathscr{J}$ under the constraint

$$
\iint_{\mathbb{R}^{d} \times \mathbb{R}^{d}} f(x, v) \mathrm{d} v \mathrm{~d} x=m .
$$

We thus introduce, for $\lambda \in \mathbb{R}, \mathscr{J}_{\lambda}=\mathscr{J}+\lambda\left(\iint_{\mathbb{R}^{d} \times \mathbb{R}^{d}} f \mathrm{~d} v \mathrm{~d} x-m\right)$. The Euler-Lagrange relations yield the following conclusions

- with $\partial_{\pi} \mathscr{J}_{\lambda}$ we simply get $\pi_{\text {eq }}=0$,

- with $\partial_{\Psi} \mathscr{J}_{\lambda}$ we obtain

$$
\sigma_{2}(z)\left(\sigma_{1} \star \int_{\mathbb{R}^{d}} \mathscr{M}_{\mathrm{eq}}(\cdot, v) \mathrm{d} v\right)(x)=c^{2} \Delta_{z} \Psi_{\mathrm{eq}}(x, z) .
$$

Denoting by $\Gamma$ the solution of $\Delta_{z} \Gamma=\sigma_{2}$, we are led to

$$
\Psi_{\mathrm{eq}}(x, z)=\frac{\Gamma(z)}{c^{2}} \sigma_{1} \star\left(\int_{\mathbb{R}^{d}} \mathscr{M}_{\mathrm{eq}}(\cdot, v) \mathrm{d} v\right)(x) .
$$

The associated equilibrium potential thus reads

$$
\begin{aligned}
\Phi_{\mathrm{eq}}(x) & =\int_{\mathbb{R}^{d}} \sigma_{1}(x-y)\left(\int_{\mathbb{R}^{n}} \sigma_{2}(z) \Psi_{\mathrm{eq}}(y, z) \mathrm{d} z\right) \mathrm{d} y \\
& =\sigma_{1} \star \sigma_{1} \star\left(\int_{\mathbb{R}^{d}} \mathscr{M}_{\mathrm{eq}}(\cdot, v) \mathrm{d} v\right)(x) \times\left(-\frac{1}{c^{2}} \int_{\mathbb{R}^{n}}\left|\nabla_{z} \Gamma(z)\right|^{2} \mathrm{~d} z\right) \\
& =-\frac{\kappa}{c^{2}} \sigma_{1} \star \sigma_{1} \star\left(\int_{\mathbb{R}^{d}} \mathscr{M}_{\mathrm{eq}}(\cdot, v) \mathrm{d} v\right)(x) .
\end{aligned}
$$


- with $\partial_{f} \mathscr{J}_{\lambda}$ we infer

$$
\begin{aligned}
h^{\prime}\left(\mathscr{M}_{\mathrm{eq}}(x, v)\right) & =-\frac{v^{2}}{2}-V(x)-\sigma_{1} \star \int_{\mathbb{R}^{n}} \sigma_{2}(z) \Psi_{\mathrm{eq}}(\cdot, z) \mathrm{d} z-\lambda_{\mathrm{eq}} \\
& =-\frac{v^{2}}{2}-V(x)+\frac{\kappa}{c^{2}} \sigma_{1} \star \sigma_{1} \star\left(\int_{\mathbb{R}^{d}} \mathscr{M}_{\mathrm{eq}}(\cdot, v) \mathrm{d} v\right)(x)-\lambda_{\mathrm{eq}},
\end{aligned}
$$

with $\lambda_{\text {eq }}$ the Lagrange multiplier associated to the mass constraint.

Therefore, the equilibrium distribution function is defined by the implicit formula

$$
\mathscr{M}_{\mathrm{eq}}(x, v)=\Upsilon\left(\frac{v^{2}}{2}+V(x)-\frac{\kappa}{c^{2}} \Sigma \star \rho_{\mathrm{eq}}(x)+\lambda_{\mathrm{eq}}\right), \quad \Sigma=\sigma_{1} \star \sigma_{1}, \quad \rho_{\mathrm{eq}}=\int_{\mathbb{R}^{d}} \mathscr{M}_{\mathrm{eq}} \mathrm{d} v
$$

where

$$
\Upsilon(s)=\left(h^{\prime}\right)^{-1}(-s),
$$

and the Lagrange multiplier $\lambda_{\text {eq }}$ is determined by

$$
\iint_{\mathbb{R}^{d} \times \mathbb{R}^{d}} \mathscr{M}_{\mathrm{eq}} \mathrm{d} v \mathrm{~d} x=m
$$

We readily check that

$$
\left(v \cdot \nabla_{x}-\nabla_{x}\left(V+\Psi_{\text {eq }}\right) \cdot \nabla_{v}\right) \mathscr{M}_{\mathrm{eq}}=0
$$

so that $\mathscr{M}_{\text {eq }}$ is a stationary solution of (1), as anticipated. This comes from the fact that, denoting $\mathscr{E}_{\text {eq }}(x, v)=\frac{v^{2}}{2}+V(x)-\frac{\kappa}{c^{2}} \Sigma \star \rho_{\text {eq }}(x)=\frac{v^{2}}{2}+V(x)+\Phi_{\text {eq }}(x), \mathscr{M}_{\text {eq }}$ can be interpreted as a function of this energy and $\left(v \cdot \nabla_{x}-\nabla_{x}\left(V+\Psi_{\text {eq }}\right) \cdot \nabla_{v}\right) \mathscr{M}_{\text {eq }}$ is the Lie bracket of $\mathscr{E}_{\text {eq }}$ and $\mathscr{M}_{\text {eq }}$. Moreover, $\Psi_{\text {eq }}$ is a stationary solution of the associated wave equation (2)-(3) while finally $\Phi_{\text {eq }}$ satisfies (4).

The specific case $h(s)=s \ln (s)-s$ leads to (6), with $\Upsilon(s)=e^{-s}, Z_{\text {eq }}=e^{-\lambda_{\text {eq }}}$. With the change of variable $v=\sqrt{2 s} \omega, s \geq 0, \omega \in \mathbb{S}^{d-1}$, we can rewrite

$$
\begin{aligned}
& \rho_{\mathrm{eq}}(x)=\mu\left(V(x)+\Phi_{\mathrm{eq}}(x)+\lambda_{\mathrm{eq}}\right), \quad \Phi_{\mathrm{eq}}=-\frac{\kappa}{c^{2}} \Sigma \star \rho_{\mathrm{eq}}, \\
& \mu(U)=2^{d / 2-1}\left|\mathbb{S}^{d-1}\right| \int_{0}^{\infty} s^{d / 2-1} \Upsilon(s+U) \mathrm{d} s .
\end{aligned}
$$

Through this approach we have therefore defined a broad class of stationary states for (1)-(4), in contrast to the case of a single particle where, as mentioned before, a unique equilibrium exists. We now investigate their stability.

Let us now detail the technical requirements on which the analysis is based. Throughout the paper we assume

a) $n \geq 3$,

b) $\sigma_{1}, \sigma_{2}$ are $C^{\infty}$, compactly supported, radially symmetric and non negative. 
Assumption a) already appears in [5] as well as in [8]. In particular, we should bear in mind that the definition of $\kappa$ uses this assumption. We refer the reader to [5] for further comments on the physical motivation for assuming $n \geq 3$. Assumption b) is certainly far from optimal, as it will appear within the discussion, but it sticks with the framework introduced and motivated in [5]. To give a precise definition of the coupling term in the energy (7), we proceed as follows. First, we define, for $\Psi: \mathbb{R}^{d} \times \mathbb{R}^{n} \rightarrow \mathbb{R}$, and $\rho: \mathbb{R}^{d} \rightarrow \mathbb{R}:$

$$
\mathscr{B}(\Psi, \rho)=\int_{\mathbb{R}^{d}}\left(\sigma_{1} \star \rho\right)(x)\left(\int_{\mathbb{R}^{n}} \sigma_{2}(z) \Psi(x, z) \mathrm{d} z\right) \mathrm{d} x .
$$

Then one has

$$
\iint_{\mathbb{R}^{d} \times \mathbb{R}^{d}} \Phi[\Psi] f \mathrm{~d} v \mathrm{~d} x=\mathscr{B}(\Psi, \rho) .
$$

Lemma 2.1 There exists a constant $C$, which does not depend on $\psi, \rho$, such that

$$
|\mathscr{B}(\Psi, \rho)| \leq C\left\|\sigma_{2}\right\|_{L^{2 n /(n+2)}\left(\mathbb{R}^{n}\right)}\left\|\sigma_{1}\right\|_{L^{2}\left(\mathbb{R}^{d}\right)}\left\|\nabla_{z} \Psi\right\|_{L^{2}\left(\mathbb{R}^{n} \times \mathbb{R}^{d}\right)}\|\rho\|_{L^{1}\left(\mathbb{R}^{d}\right)} .
$$

Proof. We shall use the Gagliardo-Nirenberg-Sobolev inequality, see e.g. [22, p. 125], [4, Theorem IX.9],

$$
\|u\|_{L^{2 n /(n-2)\left(\mathbb{R}^{n}\right)}} \leq C\|\nabla u\|_{L^{2}\left(\mathbb{R}^{n}\right)}
$$

Then, we get

$$
\begin{aligned}
& |\mathscr{B}(\Psi, \rho)| \leq\left(\int_{\mathbb{R}^{d}}\left|\int_{\mathbb{R}^{n}} \sigma_{2}(z) \Psi(x, z) \mathrm{d} z\right|^{2} \mathrm{~d} x\right)^{1 / 2}\left(\int_{\mathbb{R}^{d}}\left|\sigma_{1} \star \rho(x)\right|^{2} \mathrm{~d} x\right)^{1 / 2} \\
& \leq\left(\int_{\mathbb{R}^{d}}\left\|\sigma_{2}\right\|_{L^{2 n /(n+2)\left(\mathbb{R}^{n}\right)}}^{2}\left(\int_{\mathbb{R}^{n}}|\Psi(x, z)|^{2 n /(n-2)} \mathrm{d} z\right)^{(n-2) / n} \mathrm{~d} x\right)^{1 / 2}\left\|\sigma_{1}\right\|_{L^{2}\left(\mathbb{R}^{d}\right)}\|\rho\|_{L^{1}\left(\mathbb{R}^{d}\right)} \\
& \leq\left\|\sigma_{2}\right\|_{L^{2 n /(n+2)\left(\mathbb{R}^{n}\right)}}\left(\int_{\mathbb{R}^{d}} C^{2}\left(\int_{\mathbb{R}^{n}}\left|\nabla_{z} \Psi(x, z)\right|^{2} \mathrm{~d} z\right) \mathrm{d} x\right)^{1 / 2}\left\|\sigma_{1}\right\|_{L^{2}\left(\mathbb{R}^{d}\right)}\|\rho\|_{L^{1}\left(\mathbb{R}^{d}\right)} .
\end{aligned}
$$

We assume that the following requirements are fulfilled

(H0) For any $x \in \mathbb{R}^{d}$, we have $V(x) \geq 0$, and $\lim _{|x| \rightarrow \infty} V(x)=+\infty$.

(H1) Either $h(s)=s \ln (s)-s$ or $h:[0, \infty) \rightarrow[0, \infty)$ is a strictly convex $C^{2}$ function such that

$$
h(0)=0 \text { and } \lim _{s \rightarrow \infty} \frac{h(s)}{s}=+\infty
$$

with $h^{\prime}$ defined from $[0, \infty)$ to $\left[\eta_{0},+\infty\right)$ with $\eta_{0} \geq 0$. (That $\eta_{0}$ is non negative follows from the assumptions: $h(s)$ is non negative and vanishes for $s=0$.)

(H2) Let us define

$$
\Upsilon: s \in \mathbb{R} \longmapsto \Upsilon(s)= \begin{cases}0 & \text { if } s \geq-\eta_{0}, \\ \left(h^{\prime}\right)^{-1}(-s) & \text { if }-\infty<s \leq-\eta_{0}\end{cases}
$$


For any $a \in \mathbb{R}$, we have

$$
\Upsilon\left(\frac{v^{2}}{2}+V(x)+a\right) \in L^{1}\left(\mathbb{R}^{d} \times \mathbb{R}^{d}\right) .
$$

For $h(s)=s \ln (s)-s$, we further assume $x \mapsto e^{-\alpha V(x)} \in L^{1}\left(\mathbb{R}^{d}\right)$ for some $0<$ $\alpha<1 / 4$.

(H3) For any compact set $K \subset \mathbb{R}$, we can find a function $\mathscr{Z}_{K} \in L^{1}\left(\mathbb{R}^{d} \times \mathbb{R}^{d}\right)$ such that for any $a \in K$, we have $\left|\Upsilon^{\prime}\left(\frac{v^{2}}{2}+V(x)+a\right)\right| \leq \mathscr{Z}_{K}(x, v)$.

Assumptions (H0) is a confining assumption on the external potential, strengthened by the integrability condition in (H2). It is likely that these assumptions on $h$, that govern the shape of the equilibrium state, can be generalized, as will become apparent in the proof. With $h(s)=s \ln (s)-s$, we obtain the equilibrium function devised in [2], which is proportional to $\exp \left(-\frac{v^{2}}{2}-V(x)-\Phi_{\text {eq }}(x)\right)$, see (6), the self-consistent potential $\Phi_{\text {eq }}$ being determined by an integral equation. Another relevant example is given by $h(s)=s^{p}$, for $p>1$. Then, the equilibrium function casts as

$$
\mathscr{M}_{\mathrm{eq}}(x, v)=\left[-\frac{1}{p}\left(\frac{v^{2}}{2}+V(x)+\Phi_{\mathrm{eq}}(x)+\lambda_{\mathrm{eq}}\right)\right]_{+}^{1 /(p-1)},
$$

where the equilibirum potential $\Phi_{\text {eq }}$ is still determined by an integral equation and $\lambda_{\text {eq }} \in \mathbb{R}$ ensures the mass constraint. We shall see that $x \mapsto \Phi_{\text {eq }}(x)$ is bounded; hence, due to (Ho), we note that this equilibrium function is compactly supported.

Lemma 2.2 Suppose (H0)-(H2). Let $m>0$. For any function $\Phi$ continuous and bounded on $\mathbb{R}^{d}$, we can find $\lambda[\Phi] \in \mathbb{R}$ such that

$$
\iint_{\mathbb{R}^{d} \times \mathbb{R}^{d}} \Upsilon\left(\frac{v^{2}}{2}+V(x)+\Phi(x)+\lambda[\Phi]\right) \mathrm{d} v \mathrm{~d} x=m .
$$

Furthermore, for $c>0$, let us introduce the set

$$
\mathscr{C}=\left\{\Phi \in C^{0}\left(\mathbb{R}^{d}\right),-\frac{\kappa m}{c^{2}}\|\Sigma\|_{L^{\infty}\left(\mathbb{R}^{d}\right)} \leq \Phi(x) \leq 0 \text { for all } x \in \mathbb{R}^{d}\right\} .
$$

There exists $\mathscr{K}_{1}(c)>0$, such that $|\lambda[\Phi]| \leq \mathscr{K}_{1}(c)$ holds for any $\Phi \in \mathscr{C}$.

Proof. The function $s \mapsto \Upsilon(s)$ is strictly decreasing from $\left(-\infty,-\eta_{0}\right)$ to $(0,+\infty)$ (but it is defined from $\mathbb{R}$ to $[0, \infty))$. For $\Phi$ bounded and continuous, by (H0)-(H2), the mapping

$$
\mathbb{M}: \lambda \longmapsto \iint_{\mathbb{R}^{d} \times \mathbb{R}^{d}} \Upsilon\left(\frac{v^{2}}{2}+V(x)+\Phi(x)+\lambda\right) \mathrm{d} v \mathrm{~d} x
$$

is well defined, decreasing, continuous (by applying Lebesgue's theorem), and it satisfies

$$
\lim _{\lambda \rightarrow+\infty} \mathbb{M}(\lambda)=0, \quad \lim _{\lambda \rightarrow-\infty} \mathbb{M}(\lambda)=+\infty .
$$

The conclusion follows directly by the intermediate value theorem. 
The bound on $\Phi \mapsto \lambda[\Phi]$ follows from the following argument. According to (H1)(H2), $s \mapsto \Upsilon(s)$ is non increasing and we get, for any $\Phi \in \mathscr{C}$,

$$
\begin{aligned}
& \iint_{\mathbb{R}^{d} \times \mathbb{R}^{d}} \Upsilon\left(\frac{v^{2}}{2}+V(x)+\lambda[\Phi]\right) \mathrm{d} v \mathrm{~d} x \\
& \leq \iint_{\mathbb{R}^{d} \times \mathbb{R}^{d}} \Upsilon\left(\frac{v^{2}}{2}+V(x)+\Phi(x)+\lambda[\Phi]\right) \mathrm{d} v \mathrm{~d} x=m \\
& \quad \leq \iint_{\mathbb{R}^{d} \times \mathbb{R}^{d}} \Upsilon\left(\frac{v^{2}}{2}+V(x)-\frac{\kappa}{c^{2}}\|\Sigma\|_{L^{\infty}\left(\mathbb{R}^{d}\right)}+\lambda[\Phi]\right) \mathrm{d} v \mathrm{~d} x .
\end{aligned}
$$

If $\lambda[\Phi] \rightarrow+\infty$ (resp. $\lambda[\Phi] \rightarrow-\infty$ ), the bound from above tends to 0 (resp. the bound from below tends to $+\infty$ ), which leads to a contradiction.

Having disposed of these preliminaries, we turn to the analysis of the equation satisfied by the critical points of the functional $\mathscr{J}$ on the mass shell.

Proposition 2.3 Let $m>0$. Suppose $(\mathbf{H 0})-(\mathbf{H 3})$. There exists $c_{0}>0$ such that for any $c>c_{0}$, the equations

$$
\begin{aligned}
& \mathscr{M}_{\mathrm{eq}}(x, v)=\Upsilon\left(\frac{v^{2}}{2}+V(x)+\Phi_{\mathrm{eq}}(x)+\lambda_{\mathrm{eq}}\right), \\
& \Phi_{\mathrm{eq}}(x)=-\frac{\kappa}{c^{2}} \Sigma \star \rho_{\mathrm{eq}}, \quad \rho_{\mathrm{eq}}(x)=\int_{\mathbb{R}^{d}} \mathscr{M}_{\mathrm{eq}}(x, v) \mathrm{d} v, \\
& \iint_{\mathbb{R}^{d} \times \mathbb{R}^{d}} \mathscr{M}_{\mathrm{eq}}(x, v) \mathrm{d} v \mathrm{~d} x=\int_{\mathbb{R}^{d}} \rho_{\mathrm{eq}}(x) \mathrm{d} x=m,
\end{aligned}
$$

for the critical points of $\mathscr{J}$ with mass constraint admit a unique solution.

Proof. We consider the map

$\mathscr{T}: \Phi \in \mathscr{C} \longmapsto \mathscr{T}[\Phi]=-\frac{\kappa}{c^{2}} \Sigma \star \rho[\Phi], \quad \rho[\Phi](x)=\int_{\mathbb{R}^{d}} \Upsilon\left(\frac{v^{2}}{2}+V(x)+\Phi(x)+\lambda[\Phi]\right) \mathrm{d} v$

where $\lambda[\Phi] \in \mathbb{R}$ is such that

$$
\int_{\mathbb{R}^{d}} \rho[\Phi](x) \mathrm{d} x=m .
$$

Lemma 2.2 ensures that this definition makes sense. In fact, a direct application of the implicit function theorem (that uses (H3)) tells us that the mapping $\Phi \mapsto \lambda[\Phi]$ is $C^{1}$ and we are going to compute its derivative. Moreover, $\mathscr{T}$ maps $\mathscr{C}$ into itself. We see $\Phi_{\text {eq }}$ as a fixed point of $\mathscr{T}$ and we shall prove that $\mathscr{T}$ is a contraction provided $c$ is large enough. Let $\Phi$ and $\Phi^{\prime}$ in $\mathscr{C}$. We start with the simple observation

$$
\left|\mathscr{T}[\Phi]-\mathscr{T}\left[\Phi^{\prime}\right]\right| \leq \frac{\kappa}{c^{2}}\|\Sigma\|_{L^{\infty}\left(\mathbb{R}^{d}\right)}\left\|\rho[\Phi]-\rho\left[\Phi^{\prime}\right]\right\|_{L^{1}\left(\mathbb{R}^{d}\right)} .
$$

Writing $\Phi_{\theta}=\Phi+\theta\left(\Phi^{\prime}-\Phi\right)$ and $\lambda_{\theta}=\lambda\left[\Phi_{\theta}\right]$ for $0 \leq \theta \leq 1$, we obtain

$$
\begin{aligned}
\mid \rho & {[\Phi]-\rho\left[\Phi^{\prime}\right]|(x)=| \int_{0}^{1} \int_{\mathbb{R}^{d}} \frac{\mathrm{d}}{\mathrm{d} \theta}\left[\Upsilon\left(\frac{v^{2}}{2}+V(x)+\Phi_{\theta}(x)+\lambda_{\theta}\right)\right] \mathrm{d} v \mathrm{~d} \theta \mid } \\
& =\left|\int_{0}^{1} \int_{\mathbb{R}^{d}} \Upsilon^{\prime}\left(\frac{v^{2}}{2}+V(x)+\Phi_{\theta}(x)+\lambda_{\theta}\right)\left\{\left(\Phi^{\prime}-\Phi\right)(x)+\lambda^{\prime}\left[\Phi_{\theta}\right]\left(\Phi^{\prime}-\Phi\right)\right\} \mathrm{d} \theta \mathrm{d} v\right| .
\end{aligned}
$$


We find the (signed) measure $\lambda^{\prime}[\Phi]$ by taking the derivative with respect to $\Phi$ of the mass constraint: for any continuous and bounded function $\varphi$, we get

$$
0=\iint_{\mathbb{R}^{d} \times \mathbb{R}^{d}} \Upsilon^{\prime}\left(\frac{v^{2}}{2}+V(x)+\Phi(x)+\lambda[\Phi]\right)\left(\varphi(x)+\lambda^{\prime}[\Phi](\varphi)\right) \mathrm{d} v \mathrm{~d} x,
$$

the formula makes sense owing to (H3). Therefore, we obtain

$$
\lambda^{\prime}[\Phi](\varphi)=\frac{-\iint_{\mathbb{R}^{d} \times \mathbb{R}^{d}} \Upsilon^{\prime}\left(\frac{v^{2}}{2}+V(x)+\Phi(x)+\lambda[\Phi]\right) \varphi(x) \mathrm{d} v \mathrm{~d} x}{\iint_{\mathbb{R}^{d} \times \mathbb{R}^{d}} \Upsilon^{\prime}\left(\frac{v^{2}}{2}+V(x)+\Phi(x)+\lambda[\Phi]\right) \mathrm{d} v \mathrm{~d} x} .
$$

We deduce from this relation that

$$
\left|\lambda^{\prime}[\Phi](\varphi)\right| \leq\|\varphi\|_{L^{\infty}\left(\mathbb{R}^{d}\right)} .
$$

Let us temporarily assume that for some $c_{0}>0$, we can find a constant $\mathscr{K}\left(c_{0}\right)$ such that for any $c \geq c_{0}$ and $\Phi \in \mathscr{C}$ we have

$$
\left|\iint_{\mathbb{R}^{d} \times \mathbb{R}^{d}} \Upsilon^{\prime}\left(\frac{v^{2}}{2}+V(x)+\Phi(x)+\lambda[\Phi]\right) \mathrm{d} v \mathrm{~d} x\right| \leq \mathscr{K}\left(c_{0}\right) .
$$

Owing to (12), it follows that

$$
\left|\rho[\Phi]-\rho\left[\Phi^{\prime}\right]\right|(x) \leq 2 \mathscr{K}\left(c_{0}\right)\left\|\Phi-\Phi^{\prime}\right\|_{L^{\infty}\left(\mathbb{R}^{d}\right)}
$$

holds for any $\Phi, \Phi^{\prime} \in \mathscr{C}$ and $c \geq c_{0}$. We conclude that

$$
\left|\mathscr{T}[\Phi]-\mathscr{T}\left[\Phi^{\prime}\right]\right| \leq \frac{2 \kappa}{c^{2}} \mathscr{K}\left(c_{0}\right)\|\Sigma\|_{L^{\infty}\left(\mathbb{R}^{d}\right)}\left\|\Phi-\Phi^{\prime}\right\|_{L^{\infty}\left(\mathbb{R}^{d}\right)} .
$$

We now prove (12). As $c \rightarrow \infty$ the functions in $\mathscr{C}$ converge uniformly to 0 ; moreover, by virtue of (11), we see that $\lambda[\Phi]$ tends to $\lambda_{0} \in \mathbb{R}$, which is determined by

$$
\iint_{\mathbb{R}^{d} \times \mathbb{R}^{d}} \Upsilon\left(\frac{v^{2}}{2}+V(x)+\lambda_{0}\right) \mathrm{d} v \mathrm{~d} x=m,
$$

see Lemma 2.2. For $c>0$ and $\Phi \in \mathscr{C}$, it follows from (H3) that the integral

$$
\iint_{\mathbb{R}^{d} \times \mathbb{R}^{d}} \Upsilon^{\prime}\left(\frac{v^{2}}{2}+V(x)+\Phi(x)+\lambda[\Phi]\right) \mathrm{d} v \mathrm{~d} x
$$

is well defined. In addition, as $c \rightarrow \infty$ it tends to

$$
\iint_{\mathbb{R}^{d} \times \mathbb{R}^{d}} \Upsilon^{\prime}\left(\frac{v^{2}}{2}+V(x)+\lambda_{0}\right) \mathrm{d} v \mathrm{~d} x
$$

by continuity and the dominated convergence theorem. It allows us to check that (12) holds for $c \geq c_{0}$ large enough. Moreover we have

$$
\lim _{c \rightarrow \infty} \frac{\mathscr{K}\left(c_{0}\right)}{c^{2}}=0
$$

and $c_{0}$ can be chosen large enough so that $\mathscr{T}$ is a contraction on $\mathscr{C}$ for $c \geq c_{0}$.

We turn to the minimization problem. We extend $h$ by $+\infty$ when its argument is 
negative, so that $\mathscr{J}$ is defined over

$$
\begin{aligned}
& f \in L^{1}\left(\mathbb{R}^{d} \times \mathbb{R}^{d}\right), \quad\left(v^{2}+V(x)\right) f \in L^{1}\left(\mathbb{R}^{d} \times \mathbb{R}^{d}\right), \\
& \nabla_{z} \Psi \in L^{2}\left(\mathbb{R}^{d} \times \mathbb{R}^{n}\right), \quad \pi \in L^{2}\left(\mathbb{R}^{d} \times \mathbb{R}^{n}\right) .
\end{aligned}
$$

We wish to minimize $\mathscr{J}$ under a mass constraint; the connection with the critical points discussed in Proposition 2.3 is established through the following claim.

Lemma 2.4 Let $m>0$. If $\mathscr{J}$ admits a minimizer $(f, \Psi, \pi)$ over the functions in (13) satisfying the mass constraint $\iint_{\mathbb{R}^{d} \times \mathbb{R}^{d}} f \mathrm{~d} v \mathrm{~d} x=m$, then it coincides with the critical point $f=\mathscr{M}_{\mathrm{eq}}, \Psi=\Psi_{\mathrm{eq}}$ and $\pi=0$.

It follows from this, together with the uniqueness of the critical point, that there is at most one minimizer. That such a minimizer exists and that, therefore, the unique critical point is indeed a minimizer, will be proven below, in Lemma 3.3.

Proof. By virtue of Lemma $2.1 \mathscr{J}$ is bounded from below, when working on non negative functions $f$ with fixed total mass $m>0$ (see the manipulation in Appendix A). Let $(f, \Psi, \pi)$ be a minimizer of $\mathscr{J}$ with the constraint $\iint_{\mathbb{R}^{d} \times \mathbb{R}^{d}} f \mathrm{~d} v \mathrm{~d} x=m$. Since $\mathscr{J}(f, \Psi, \pi)$ is finite, we deduce that $f(x, v) \geq 0$ a. e. and $h(f) \in L^{1}\left(\mathbb{R}^{d} \times \mathbb{R}^{d}\right)$. We readily check that $\pi=0$ and $-\Delta_{z} \Psi(x, z)=-\sigma_{2}(z)\left(\sigma_{1} \star \int_{\mathbb{R}^{d}} f(\cdot, v) \mathrm{d} v\right)(x)$, just by expanding $\mathscr{J}(f, \Psi+\delta \psi, \pi)-\mathscr{J}(f, \Psi, \pi) \geq 0$ and $\mathscr{J}(f, \Psi, \pi+\delta \widetilde{\pi})-\mathscr{J}(f, \Psi, \pi) \geq 0$ with $\psi, \tilde{\pi} \in C_{c}^{\infty}\left(\mathbb{R}^{d} \times \mathbb{R}^{n}\right)$, and letting $\delta$ go to 0 . It remains to find the expression of the distribution function $f$. We follow the arguments detailed in $[19,23]$. We start by discussing the case where $h$ is a $C^{1}$ function over $[0, \infty)$. For $\epsilon>0$, we set

$$
S_{\epsilon}=\left\{(x, v) \in \mathbb{R}^{d} \times \mathbb{R}^{d}, \epsilon \leq f(x, v) \leq 1 / \epsilon\right\} .
$$

We consider $w \in L^{\infty}\left(\mathbb{R}^{d} \times \mathbb{R}^{d}\right)$, with $\operatorname{supp}(w) \subset B(0, R)$, for some $0<R<\infty$ and such that $w \geq 0$ on $\complement S_{\epsilon}$. As a consequence of the mass constraint, and the BienayméTchebychev inequality, we have, for small enough $\epsilon$ 's,

$$
0<\operatorname{meas}\left(S_{\epsilon}\right) \leq \frac{m}{\epsilon}<\infty
$$

Then, we can find $\tau(\epsilon)>0$ such that, for any $0<\tau \leq \tau(\epsilon)$ the function

$$
f_{\tau}(x, v)=f(x, v)+\tau\left(w(x, v)-\mathbf{1}_{S_{\epsilon}}(x, v) \frac{\int_{\mathbb{R}^{d} \times \mathbb{R}^{d}} w(y, \xi) \mathrm{d} y \mathrm{~d} \xi}{\operatorname{meas}\left(S_{\epsilon}\right)}\right)
$$

is non-negative and satisfies $\iint_{\mathbb{R}^{d} \times \mathbb{R}^{d}} f_{\tau} \mathrm{d} v \mathrm{~d} x=m$. Accordingly we have

$$
\mathscr{J}\left(f_{\tau}, \Psi, \pi\right)-\mathscr{J}(f, \Psi, \pi) \geq 0 .
$$

Since $h$ is $C^{1}$ and $f_{\tau}-f$ is supported on a set of finite measure, it follows that

$$
\begin{aligned}
& \iint_{\mathbb{R}^{d} \times \mathbb{R}^{d}}\left(h^{\prime}(f(x, v))+\frac{v^{2}}{2}+V(x)+\Phi[\Psi](x)\right)\left(w(x, v)-\mathbf{1}_{S_{\epsilon}}(x, v) \frac{\int_{\mathbb{R}^{d} \times \mathbb{R}^{d}} w(y, \xi) \mathrm{d} y \mathrm{~d} \xi}{\operatorname{meas}\left(S_{\epsilon}\right)}\right) \mathrm{d} v \mathrm{~d} x \geq 0 \\
& \times(w)
\end{aligned}
$$


which recasts as

$$
\iint_{\mathbb{R}^{d} \times \mathbb{R}^{d}} w(x, v)\left(h^{\prime}(f(x, v))+\frac{v^{2}}{2}+V(x)+\Phi[\Psi](x)+\lambda_{\epsilon}\right) \mathrm{d} v \mathrm{~d} x \geq 0
$$

where we have set

$$
\lambda_{\epsilon}=-\frac{1}{\operatorname{meas}\left(S_{\epsilon}\right)} \int_{S_{\epsilon}}\left(h^{\prime}(f(y, \xi))+\frac{\xi^{2}}{2}+V(y)+\Phi[\Psi](y)\right) \mathrm{d} y \mathrm{~d} \xi \in \mathbb{R} .
$$

Since this relation holds for any trial function $w$ verifying the conditions prescribed above, we deduce the following properties:

- On the one hand, for a. e. $(x, v) \in S_{\epsilon}$, we have

$$
h^{\prime}(f(x, v))+\frac{v^{2}}{2}+V(x)+\Phi[\Psi](x)=-\lambda_{\epsilon}
$$

which, additionally, tells us that $\lambda_{\epsilon}=\lambda$ does not depend on $\epsilon$.

- On the other hand, on $\complement S_{\epsilon}$, we get $h^{\prime}(f(x, v))+\frac{v^{2}}{2}+V(x)+\Phi[\Psi](x)+\lambda \geq 0$.

Letting $\epsilon$ go to 0 , we conclude that either $f(x, v)>0$, and $h^{\prime}(f(x, v))=-\frac{v^{2}}{2}-V(x)-$ $\Phi[\Psi](x)-\lambda>\eta_{0}$, or $f(x, v)=0$ and $\frac{v^{2}}{2}+V(x)+\Phi[\Psi](x)+\lambda \geq-\eta_{0}$. We recover the formula for the critical points of $\mathscr{J}$.

The argument needs to be slightly adapted to handle the case $h(s)=s \ln (s)-s$, for which $h^{\prime}(0)$ is not defined. We therefore need to exclude the possibility that $f(x, v)=0$. The proof therefore begins with the justification that $f$ takes strictly positive values only. We argue by contradiction: suppose that the set $\mathscr{O}=\left\{(x, v) \in \mathbb{R}^{d} \times \mathbb{R}^{d}, f(x, v)=\right.$ $0\}$ has a positive measure (possibly $+\infty$ ). The regularity of the Lebesgue measure allows us to find a compact set $K \subset \mathscr{O}$ such that meas $(K)>0$. We now set for $\tau>0$

$$
f_{\tau}(x, v)=f(x, v)+\tau\left(\mathbf{1}_{K}-\mathbf{1}_{S_{\epsilon}} \frac{\operatorname{meas}(K)}{\operatorname{meas}\left(S_{\epsilon}\right)}\right),
$$

where $S_{\epsilon}$ is defined as above. Clearly $K \cap S_{\epsilon}=\emptyset$ and, again, we can find $\tau(\epsilon)>0$ such that $f_{\tau}$ remains non negative for any $0<\tau \leq \tau(\epsilon)$, while $\iint_{\mathbb{R}^{d} \times \mathbb{R}^{d}} f_{\tau} \mathrm{d} v \mathrm{~d} x=m$. Therefore, we have

$$
\begin{aligned}
0 \leq & \mathscr{J}\left(f_{\tau}, \Psi, \pi\right)-\mathscr{J}(f, \Psi, \pi) \\
\leq & \iint_{\mathbb{R}^{d} \times \mathbb{R}^{d}}\left(h\left(f_{\tau}\right)-h(f)\right) \mathrm{d} v \mathrm{~d} x \\
& +\tau \iint_{\mathbb{R}^{d} \times \mathbb{R}^{d}}\left(\frac{v^{2}}{2}+V(x)+\Phi[\Psi](x)\right)\left(\mathbf{1}_{K}-\mathbf{1}_{S_{\epsilon}} \frac{\operatorname{meas}(K)}{\operatorname{meas}\left(S_{\epsilon}\right)}\right) \mathrm{d} v \mathrm{~d} x .
\end{aligned}
$$

By definition $f_{\tau}(x, v)=f(x, v)$ on $\complement\left(K \cup S_{\epsilon}\right), f_{\tau}(x, v)=f(x, v)+\tau=\tau$ on $K$ and $f_{\tau}(x, v)=f(x, v)-\tau \frac{\operatorname{meas}(K)}{\operatorname{meas}\left(S_{\epsilon}\right)}$ on $S_{\epsilon}$. This yields

$$
\begin{aligned}
0 \leq & \operatorname{meas}(K)(\tau \ln (\tau)-\tau)+\tau \iint_{K}\left(\frac{v^{2}}{2}+V(x)+\Phi[\Psi](x)\right) \mathrm{d} v \mathrm{~d} x \\
& +\iint_{S_{\epsilon}}\left(h\left(f_{\tau}\right)-h(f)\right) \mathrm{d} v \mathrm{~d} x-\tau \frac{\operatorname{meas}(K)}{\operatorname{meas}\left(S_{\epsilon}\right)} \iint_{S_{\epsilon}}\left(\frac{v^{2}}{2}+V(x)+\Phi[\Psi](x)\right) \mathrm{d} v \mathrm{~d} x .
\end{aligned}
$$


Let us use a shorthand notation for the real number

$A=\iint_{K}\left(\frac{v^{2}}{2}+V(x)+\Phi[\Psi](x)\right) \mathrm{d} v \mathrm{~d} x-\frac{\operatorname{meas}(K)}{\operatorname{meas}\left(S_{\epsilon}\right)} \iint_{S_{\epsilon}}\left(\frac{v^{2}}{2}+V(x)+\Phi[\Psi](x)\right) \mathrm{d} v \mathrm{~d} x$.

Dividing the previous relation by $\tau>0$, we arrive at

$$
0 \leq \operatorname{meas}(K)(\ln (\tau)-1)+A+\iint_{S_{\epsilon}} \frac{h\left(f_{\tau}\right)-h(f)}{\tau} \mathrm{d} v \mathrm{~d} x
$$

As $\tau$ tends to 0 the last term tends to $\iint_{S_{\epsilon}} \ln (f) \mathrm{d} v \mathrm{~d} x$, which is also finite for any fixed $\epsilon>0$. We are led to a contradiction since $\lim _{\tau \rightarrow 0} \ln (\tau)=-\infty$. We conclude that meas $(\mathscr{O})=0$. From here, we can repeat the previous argument to identify $f$ by means of the Lagrange multiplier and to recover this way the formula that defines the critical point.

\section{Dynamical stability}

To start with, it is convenient to make the relative entropy with respect to the equilibrium state appear. For $h$ a given strictly convex function, we set

$$
H(f \mid g)=\iint_{\mathbb{R}^{d} \times \mathbb{R}^{d}}\left(h(f)-h(g)-h^{\prime}(g)(f-g)\right) \mathrm{d} v \mathrm{~d} x .
$$

This quantity measures how far $f$ is from $g$ since we have

$$
H(f \mid g) \geq 0 \text { and } H(f \mid g)=0 \text { iff } f=g .
$$

Remark 3.1 It is worth pointing out that, for the case of $h(s)=s \ln (s)-s$, the functional $H$ controls the $L^{1}$ norm of $f-g$, by virtue of the Cszisar-Kullback inequality [7, 20]. For other entropies $h$, it is possible to deduce $L^{p}$ estimates, see [6].

In the specific case where $g=\mathscr{M}_{\text {eq }}$, we can use

$$
h^{\prime}\left(\mathscr{M}_{\mathrm{eq}}(x, v)\right)=-\frac{v^{2}}{2}-V(x)-\Phi_{\mathrm{eq}}(x)-\lambda_{\mathrm{eq}}
$$

and we arrive at

$$
H\left(f \mid \mathscr{M}_{\mathrm{eq}}\right)=\iint_{\mathbb{R}^{d} \times \mathbb{R}^{d}}\left(h(f)-h\left(\mathscr{M}_{\mathrm{eq}}\right)+\left(f-\mathscr{M}_{\mathrm{eq}}\right)\left(\frac{v^{2}}{2}+V(x)+\Phi_{\mathrm{eq}}(x)\right)\right) \mathrm{d} v \mathrm{~d} x
$$

provided

$$
\iint_{\mathbb{R}^{d} \times \mathbb{R}^{d}} f \mathrm{~d} v \mathrm{~d} x=\iint_{\mathbb{R}^{d} \times \mathbb{R}^{d}} \mathscr{M}_{\mathrm{eq}} \mathrm{d} v \mathrm{~d} x=m
$$

We now compute

$$
\mathscr{J}(f, \Psi, \pi)-\mathscr{J}\left(\mathscr{M}_{\mathrm{eq}}, \Psi_{\mathrm{eq}}, \pi_{\mathrm{eq}}\right)=I+I I+\frac{1}{2} \iint_{\mathbb{R}^{d} \times \mathbb{R}^{n}}|\pi|^{2} \mathrm{~d} z \mathrm{~d} x
$$


with, on the one hand

$$
\begin{aligned}
I & =\iint_{\mathbb{R}^{d} \times \mathbb{R}^{d}}\left(h(f)+\left(\frac{v^{2}}{2}+V(x)\right) f-h\left(\mathscr{M}_{\mathrm{eq}}\right)-\left(\frac{v^{2}}{2}+V(x)\right) \mathscr{M}_{\mathrm{eq}}\right) \mathrm{d} v \mathrm{~d} x \\
& =H\left(f \mid \mathscr{M}_{\mathrm{eq}}\right)+\iint_{\mathbb{R}^{d} \times \mathbb{R}^{d}}\left(\mathscr{M}_{\mathrm{eq}}-f\right) \Phi_{\mathrm{eq}} \mathrm{d} v \mathrm{~d} x
\end{aligned}
$$

and, on the other hand

$$
\begin{aligned}
I I= & \iint_{\mathbb{R}^{d} \times \mathbb{R}^{d}}\left(\Phi[\Psi] f-\Phi_{\mathrm{eq}} \mathscr{M}_{\mathrm{eq}}\right) \mathrm{d} v \mathrm{~d} x+\frac{c^{2}}{2} \iint_{\mathbb{R}^{d} \times \mathbb{R}^{n}}\left(\left|\nabla_{z} \Psi\right|^{2}-\left|\nabla_{z} \Psi_{\mathrm{eq}}\right|^{2}\right) \mathrm{d} z \mathrm{~d} x \\
= & \iint_{\mathbb{R}^{d} \times \mathbb{R}^{d}} \Phi_{\mathrm{eq}}\left(f-\mathscr{M}_{\mathrm{eq}}\right) \mathrm{d} v \mathrm{~d} x+\iint_{\mathbb{R}^{d} \times \mathbb{R}^{d}} f\left(\Phi[\Psi]-\Phi_{\mathrm{eq}}\right) \mathrm{d} v \mathrm{~d} x \\
& +\frac{c^{2}}{2} \iint_{\mathbb{R}^{d} \times \mathbb{R}^{n}}\left|\nabla_{z}\left(\Psi-\Psi_{\mathrm{eq}}\right)\right|^{2} \mathrm{~d} z \mathrm{~d} x \\
& -c^{2} \iint_{\mathbb{R}^{d} \times \mathbb{R}^{n}}\left|\nabla_{z} \Psi_{\mathrm{eq}}\right|^{2} \mathrm{~d} z \mathrm{~d} x+c^{2} \iint_{\mathbb{R}^{d} \times \mathbb{R}^{n}} \nabla_{z} \Psi \cdot \nabla_{z} \Psi_{\mathrm{eq}} \mathrm{d} z \mathrm{~d} x \\
= & \iint_{\mathbb{R}^{d} \times \mathbb{R}^{d}} \Phi_{\mathrm{eq}}\left(f-\mathscr{M}_{\mathrm{eq}}\right) \mathrm{d} v \mathrm{~d} x+\iint_{\mathbb{R}^{d} \times \mathbb{R}^{d}} f \sigma_{1} \star\left(\int_{\mathbb{R}^{n}} \sigma_{2}\left(\Psi-\Psi_{\mathrm{eq}}\right) \mathrm{d} z\right) \mathrm{d} v \mathrm{~d} x \\
& +\frac{c^{2}}{2} \iint_{\mathbb{R}^{d} \times \mathbb{R}^{n}}\left|\nabla_{z}\left(\Psi-\Psi_{\mathrm{eq}}\right)\right|^{2} \mathrm{~d} z \mathrm{~d} x+c^{2} \iint_{\mathbb{R}^{d} \times \mathbb{R}^{n}}\left(\Psi_{\mathrm{eq}}-\Psi\right) \cdot \Delta_{y} \Psi_{\mathrm{eq}} \mathrm{d} z \mathrm{~d} x \\
= & \iint_{\mathbb{R}^{d} \times \mathbb{R}^{d}} \Phi_{\mathrm{eq}}\left(f-\mathscr{M}_{\mathrm{eq}}\right) \mathrm{d} v \mathrm{~d} x+\iint_{\mathbb{R}^{d} \times \mathbb{R}^{d}} f \sigma_{1} \star\left(\int_{\mathbb{R}^{n}} \sigma_{2}\left(\Psi-\Psi_{\mathrm{eq}}\right) \mathrm{d} z\right) \mathrm{d} v \mathrm{~d} x \\
& +\frac{c^{2}}{2} \iint_{\mathbb{R}^{d} \times \mathbb{R}^{n}}\left|\nabla_{z}\left(\Psi-\Psi_{\mathrm{eq}}\right)\right|^{2} \mathrm{~d} z \mathrm{~d} x+c^{2} \iint_{\mathbb{R}^{d} \times \mathbb{R}^{n}}\left(\Psi_{\mathrm{eq}}-\Psi\right) \sigma_{2} \sigma_{1} \star\left(\int_{\mathbb{R}^{d}} \mathscr{M} \mathrm{eq} \mathrm{d} v\right) \mathrm{d} z \mathrm{~d} x \\
= & \iint_{\mathbb{R}^{d} \times \mathbb{R}^{d}} \Phi_{\mathrm{eq}}\left(f-\mathscr{M}_{\mathrm{eq}}\right) \mathrm{d} v \mathrm{~d} x+\frac{c^{2}}{2} \iint_{\mathbb{R}^{d} \times \mathbb{R}^{n}}\left|\nabla_{z}\left(\Psi-\Psi_{\mathrm{eq}}\right)\right|^{2} \mathrm{~d} z \mathrm{~d} x \\
& +\iiint_{\mathbb{R}^{d} \times \mathbb{R}^{d} \times \mathbb{R}^{n}} \sigma_{2}\left(\Psi-\Psi_{\mathrm{eq}}\right) \sigma_{1} \star\left(f-\mathscr{M}_{\mathrm{eq}}\right) \mathrm{d} v \mathrm{~d} z \mathrm{~d} x .
\end{aligned}
$$

We end up with

$$
\begin{aligned}
& \mathscr{J}(f, \Psi, \pi)-\mathscr{J}\left(\mathscr{M}_{\mathrm{eq}}, \Psi_{\mathrm{eq}}, \pi_{\mathrm{eq}}\right) \\
& =H\left(f \mid \mathscr{M}_{\mathrm{eq}}\right)+\frac{c^{2}}{2} \iint_{\mathbb{R}^{d} \times \mathbb{R}^{n}}\left|\nabla_{z}\left(\Psi-\Psi_{\mathrm{eq}}\right)\right|^{2} \mathrm{~d} z \mathrm{~d} x+\frac{1}{2} \iint_{\mathbb{R}^{d} \times \mathbb{R}^{n}}|\pi|^{2} \mathrm{~d} z \mathrm{~d} x \\
& \quad+\iiint_{\mathbb{R}^{d} \times \mathbb{R}^{d} \times \mathbb{R}^{n}} \sigma_{2}(z)\left(\Psi-\Psi_{\mathrm{eq}}\right)(x, z) \sigma_{1} \star\left(f-\mathscr{M}_{\mathrm{eq}}\right)(x, v) \mathrm{d} v \mathrm{~d} z \mathrm{~d} x .
\end{aligned}
$$

The first three terms in the right hand side are non negative; only the last integral, which is nothing but

$$
\mathscr{B}\left(\Psi-\Psi_{\mathrm{eq}}, \int_{\mathbb{R}^{d}} f-\mathscr{M}_{\mathrm{eq}} \mathrm{d} v\right),
$$

does not have a definite sign. The main result of this paper can be stated as follows. 
Theorem 3.2 Let $m>0$ and suppose $(\mathbf{H 0})-(\mathbf{H 3})$. Let $c>c_{0}$, as in Proposition 2.3. For any $\epsilon>0$, there exists $\eta>0$ such that, if the initial data

$$
\left.f\right|_{t=0}=f_{0},\left.\quad \Psi\right|_{t=0}=\Psi_{0},\left.\quad \partial_{t} \Psi\right|_{t=0}=\Psi_{1}
$$

for (1)-(4) satisfies

$$
H\left(f_{0} \mid \mathscr{M}_{\mathrm{eq}}\right)+\frac{c^{2}}{2} \iint_{\mathbb{R}^{d} \times \mathbb{R}^{n}}\left|\nabla_{z}\left(\Psi_{0}-\Psi_{\mathrm{eq}}\right)\right|^{2} \mathrm{~d} z \mathrm{~d} x+\frac{1}{2} \iint_{\mathbb{R}^{d} \times \mathbb{R}^{n}}\left|\Psi_{1}\right|^{2} \mathrm{~d} z \mathrm{~d} x \leq \eta
$$

then, for any $t \geq 0$, we have

$$
\begin{aligned}
& H\left(f(t, \cdot) \mid \mathscr{M}_{\mathrm{eq}}\right) \\
& +\frac{c^{2}}{2} \iint_{\mathbb{R}^{d} \times \mathbb{R}^{n}}\left|\nabla_{z}\left(\Psi(t, x, z)-\Psi_{\mathrm{eq}}(x, z)\right)\right|^{2} \mathrm{~d} z \mathrm{~d} x+\frac{1}{2} \iint_{\mathbb{R}^{d} \times \mathbb{R}^{n}}\left|\partial_{t} \Psi(t, x, z)\right|^{2} \mathrm{~d} z \mathrm{~d} x \leq \epsilon .
\end{aligned}
$$

As explained in [5], the condition that the propagation speed $c$ of the waves must be large can be understood to mean that the environment can evacuate energy quickly to infinity. Whether this condition is necessary for the stability result above is not clear. It is not needed in [5] to prove stability of a single particle coupled to a vibrating environment in the presence of a confining potential. But, as pointed out before, the situation here is more complex. Even the existence-uniqueness of the equilibria we study here is guaranteed only for large enough $c$ (see Proposition 2.3 and [2, Theorem 2.1] about the issue of the uniqueness of the equilibria with prescribed mass). The main ingredient for proving Theorem 3.2 is the following characterization of the minimizers of $\mathscr{J}$, which completes Lemma 2.4.

Lemma 3.3 (Minimization of $\mathscr{J}$ ) The following assertions hold:

(i) The functional $\mathscr{J}$ has a unique minimizer over (13) with a particle distribution function with mass $m$ and it is $\left(\mathscr{M}_{\mathrm{eq}}, \Psi_{\mathrm{eq}}, \pi_{\mathrm{eq}}\right)$.

(ii) Let $\left(\left(g^{\nu}, \Psi^{\nu}, \pi^{\nu}\right)\right)_{\nu \in \mathbb{N}}$ be a minimizing sequence of the functional $\mathscr{J}$, among the distributions with mass $m$; then, we can extract a subsequence $\left(\nu_{k}\right)_{k \in \mathbb{N}}$ such that $\left(g^{\nu_{k}}, \Psi^{\nu_{k}}, \pi^{\nu_{k}}\right)$ converges weakly to $\left(\mathscr{M}_{\mathrm{eq}}, \Psi_{\mathrm{eq}}, \pi_{\mathrm{eq}}\right)$ and we have

$$
\lim _{k \rightarrow \infty} \iiint_{\mathbb{R}^{d} \times \mathbb{R}^{d} \times \mathbb{R}^{n}} \sigma_{2}(z)\left(\Psi^{\nu_{k}}-\Psi_{\mathrm{eq}}\right)(x, z) \sigma_{1} \star\left(g^{\nu_{k}}-\mathscr{M}_{\mathrm{eq}}\right)(x, v) \mathrm{d} v \mathrm{~d} z \mathrm{~d} x=0 .
$$

Proof. We have seen that $\left(\mathscr{M}_{\text {eq }}, \Psi_{\text {eq }}, \pi_{\text {eq }}\right)$ is the unique critical point of $\mathscr{J}$ among the distributions with mass $m$ (see Proposition 2.3). By Lemma 2.4, if $\mathscr{J}$ has a minimizer it has to be $\left(\mathscr{M}_{\text {eq }}, \Psi_{\text {eq }}, \pi_{\text {eq }}\right)$. In order to prove $(i)$, we just have to prove that $\mathscr{J}$ admits at least one minimizer.

To this end, let us consider $\left(\left(g^{\nu}, \Psi^{\nu}, \pi^{\nu}\right)\right)_{\nu \in \mathbb{N}}$, a minimizing sequence of $\mathscr{J}$. As explained in Appendix A, it follows from the boundedness of the functional $\mathscr{J}$, together with the fact that the coupling term is bounded (see Lemma 2.1), that we can suppose, for a suitable subsequence,

- $g^{\nu_{k}} \rightarrow g$ weakly in $L^{1}\left(\mathbb{R}^{d} \times \mathbb{R}^{d}\right)$, 
- $\nabla_{z} \Psi^{\nu_{k}} \rightarrow \nabla_{z} \Psi$ weakly in $L^{2}\left(\mathbb{R}^{d} \times \mathbb{R}^{n}\right)$,

- $\pi^{\nu_{k}} \rightarrow \pi$ weakly in $L^{2}\left(\mathbb{R}^{d} \times \mathbb{R}^{n}\right)$.

In fact, the weak compactness of $\left(g^{\nu}\right)_{\nu \in \mathbb{N}}$ in $L^{1}$ follows from an application of the Dunford-Pettis theorem, see [16, Section 7.3.2], by using (H0) and (H1). This is direct when $h$ is non negative; for $h(s)=s \ln (s)-s$, the necessary estimate relies on a standard trick which is detailed in Appendix A.

We are going to prove that the limit $(g, \Psi, \pi)$ minimizes $\mathscr{J}$. It is convenient to work with the expression of $\mathscr{J}$ in (14). Indeed, owing to convexity properties (see for instance [4, Corollary III.8]), we already get

$$
\begin{gathered}
\liminf _{k \rightarrow \infty}\left\{H\left(g^{\nu_{k}} \mid \mathscr{M}_{\mathrm{eq}}\right)+\frac{c^{2}}{2} \iint_{\mathbb{R}^{d} \times \mathbb{R}^{n}}\left|\nabla_{z}\left(\Psi^{\nu_{k}}-\Psi_{\mathrm{eq}}\right)\right|^{2} \mathrm{~d} z \mathrm{~d} x+\frac{1}{2} \iint_{\mathbb{R}^{d} \times \mathbb{R}^{n}}\left|\pi_{\nu_{k}}\right|^{2} \mathrm{~d} z \mathrm{~d} x\right\} \\
\geq H\left(g \mid \mathscr{M}_{\mathrm{eq}}\right)+\frac{c^{2}}{2} \iint_{\mathbb{R}^{d} \times \mathbb{R}^{n}}\left|\nabla_{z}\left(\Psi-\Psi_{\mathrm{eq}}\right)\right|^{2} \mathrm{~d} z \mathrm{~d} x+\frac{1}{2} \iint_{\mathbb{R}^{d} \times \mathbb{R}^{n}}|\pi|^{2} \mathrm{~d} z \mathrm{~d} x .
\end{gathered}
$$

We also have

$$
\begin{aligned}
& \rho^{\nu_{k}}(x)=\int_{\mathbb{R}^{d}} g^{\nu_{k}}(x, v) \mathrm{d} v \rightarrow \rho(x)=\int_{\mathbb{R}^{d}} g(x, v) \mathrm{d} v \text { weakly in } L^{1}\left(\mathbb{R}^{d}\right) \text { as } k \rightarrow \infty, \\
& \iint_{\mathbb{R}^{d} \times \mathbb{R}^{d}} g^{\nu_{k}}(x, v) \mathrm{d} v \mathrm{~d} x=m=\iint_{\mathbb{R}^{d} \times \mathbb{R}^{d}} g(x, v) \mathrm{d} v \mathrm{~d} x .
\end{aligned}
$$

Accordingly, we observe that, for any $\varphi \in L^{\infty}\left(\mathbb{R}^{d}\right)$,

$$
\lim _{k \rightarrow \infty} \int_{\mathbb{R}^{d}} \sigma_{1} \star \rho^{\nu_{k}} \varphi \mathrm{d} x=\lim _{k \rightarrow \infty} \int_{\mathbb{R}^{d}} \rho^{\nu_{k}} \sigma_{1} \star \varphi \mathrm{d} x=\int_{\mathbb{R}^{d}} \rho \sigma_{1} \star \varphi \mathrm{d} x=\int_{\mathbb{R}^{d}} \sigma_{1} \star \rho \varphi \mathrm{d} x,
$$

since, with $\sigma_{1} \in L^{1} \cap L^{\infty}\left(\mathbb{R}^{d}\right), \sigma_{1} \star \varphi$ belongs to $L^{\infty}\left(\mathbb{R}^{d}\right)$. It means that $\sigma_{1} \star \rho^{\nu_{k}}$ converges weakly to to $\sigma_{1} \star \rho$ in $L^{1}\left(\mathbb{R}^{d}\right)$. Furthermore, since for a. e. $x \in \mathbb{R}^{d}$, the function $y \mapsto \sigma_{1}(x-y)$ lies in $L^{\infty}\left(\mathbb{R}^{d}\right)$, the convergence $\sigma_{1} \star \rho^{\nu_{k}}(x) \rightarrow \sigma_{1} \star \rho(x)$ also holds for a. e. $x \in \mathbb{R}^{d}$. Combining the two informations we deduce that $\sigma_{1} \star$ $\rho^{\nu_{k}}$ converges strongly to $\sigma_{1} \star \rho$ in $L^{1}\left(\mathbb{R}^{d}\right)$ see $\left[16\right.$, Theorem 7.60]. Since $\sigma_{1} \in L^{1} \cap$ $L^{\infty}\left(\mathbb{R}^{d}\right), \sigma_{1} \star \rho^{\nu_{k}}$ is actually bounded in any $L^{p}\left(\mathbb{R}^{d}\right)$ spaces, $1 \leq p \leq \infty$, and the convergence holds strongly in $L^{p}\left(\mathbb{R}^{d}\right)$ for any finite $p$. We turn now to the behavior of $\int_{\mathbb{R}^{n}} \sigma_{2}(z) \Psi^{\nu_{k}}(x, z) \mathrm{d} z$. The Gagliardo-Nirenberg-Sobolev inequality, that we already used for proving Lemma 2.1, tells us that $\Psi^{\nu_{k}}$ is bounded in $L^{2}\left(\mathbb{R}^{d} ; L^{2 n /(n-2)}\left(\mathbb{R}^{n}\right)\right)$. Hence, applying [15, Theorem 8.20.5] and the Banach-Alaoglu-Bourbaki theorem, we can assume that it tends to $\psi$, weakly in this space. Therefore, for any $\varphi \in L^{2}\left(\mathbb{R}^{d}\right)$, the product $\sigma_{2}(z) \varphi(x)$ lies in $L^{2}\left(\mathbb{R}^{d} ; L^{2 n /(n+2)}\left(\mathbb{R}^{n}\right)\right)$ and we get

$$
\lim _{k \rightarrow \infty} \int_{\mathbb{R}^{d}} \varphi(x)\left(\int_{\mathbb{R}^{n}} \sigma_{2}(z) \Psi^{\nu_{k}}(x, z) \mathrm{d} z\right) \mathrm{d} x=\int_{\mathbb{R}^{d}} \varphi(x)\left(\int_{\mathbb{R}^{n}} \sigma_{2}(z) \Psi(x, z) \mathrm{d} z\right) \mathrm{d} x .
$$

In other words, $\int_{\mathbb{R}^{n}} \sigma_{2}(z) \Psi^{\nu_{k}}(x, z) \mathrm{d} z$ converges to $\int_{\mathbb{R}^{n}} \sigma_{2}(z) \Psi(x, z) \mathrm{d} z$ weakly in $L^{2}\left(\mathbb{R}^{d}\right)$. We conclude that

$$
\begin{array}{r}
\lim _{k \rightarrow \infty} \iiint_{\mathbb{R}^{d} \times \mathbb{R}^{d} \times \mathbb{R}^{n}} \sigma_{2}\left(\Psi^{\nu_{k}}-\Psi_{\mathrm{eq}}\right)(x, z) \sigma_{1} \star\left(g^{\nu_{k}}-\mathscr{M}_{\mathrm{eq}}\right)(x,, v) \mathrm{d} v \mathrm{~d} z \mathrm{~d} x \\
\quad=\iiint_{\mathbb{R}^{d} \times \mathbb{R}^{d} \times \mathbb{R}^{n}} \sigma_{2}\left(\Psi-\Psi_{\mathrm{eq}}\right)(x, z) \sigma_{1} \star\left(g-\mathscr{M}_{\mathrm{eq}}\right)(x, v) \mathrm{d} v \mathrm{~d} z \mathrm{~d} x .
\end{array}
$$


We combine this relation to (15) and going back to (14) we deduce that

$$
\liminf _{k \rightarrow \infty} \mathscr{J}\left(g^{\nu_{k}}, \Psi^{\nu_{k}}, \pi^{\nu_{k}}\right) \geq \mathscr{J}(g, \Psi, \pi) .
$$

Since $\left(\left(g^{\nu}, \Psi^{\nu}, \pi^{\nu}\right)\right)_{\nu \in \mathbb{N}}$ is a minimizing sequence of $\mathscr{J}$, we deduce that $(g, \Psi, \pi)$ is a minimizer of $\mathscr{J}$, with $g$ verifying $\iint_{\mathbb{R}^{d} \times \mathbb{R}^{d}} g \mathrm{~d} v \mathrm{~d} x=m$. This already proves $(i)$. As said above, according to Proposition 2.3 and Lemma 2.4, we get

$$
g=\mathscr{M}_{\mathrm{eq}}, \quad \psi=\Psi_{\mathrm{eq}}, \quad \pi=\pi_{\text {eq }}=0 .
$$

Going back to (16), we obtain

$$
\lim _{k \rightarrow \infty} \iiint_{\mathbb{R}^{d} \times \mathbb{R}^{d} \times \mathbb{R}^{n}} \sigma_{2}(z)\left(\Psi^{\nu_{k}}-\Psi_{\mathrm{eq}}\right)(x, z) \sigma_{1} \star\left(g^{\nu_{k}}-\mathscr{M}_{\mathrm{eq}}\right)(x, v) \mathrm{d} v \mathrm{~d} z \mathrm{~d} x=0
$$

It ends the proof of $(i i)$.

Proof of Theorem 3.2. We argue by contradiction. We thus assume that we can find:

- a sequence of initial data $\left(\left(f_{0}^{\nu}, \Psi_{0}^{\nu}, \Psi_{1}^{\nu}\right)\right)_{\nu \in \mathbb{N}}$ such that

$$
\iint_{\mathbb{R}^{d} \times \mathbb{R}^{d}} f_{0}^{\nu} \mathrm{d} v \mathrm{~d} x=m
$$

and

$$
\lim _{\nu \rightarrow \infty}\left\{H\left(f_{0}^{\nu} \mid \mathscr{M}_{\mathrm{eq}}\right)+\frac{c^{2}}{2} \iint_{\mathbb{R}^{d} \times \mathbb{R}^{n}}\left|\nabla_{z}\left(\Psi_{0}^{\nu}-\Psi_{\mathrm{eq}}\right)\right|^{2} \mathrm{~d} z \mathrm{~d} x+\frac{1}{2} \iint_{\mathbb{R}^{d} \times \mathbb{R}^{n}}\left|\Psi_{1}^{\nu}\right|^{2} \mathrm{~d} z \mathrm{~d} x\right\}=0
$$

- a positive number $\epsilon>0$ and a sequence of times $\left(t^{\nu}\right)_{\nu \in \mathbb{N}}$

such that $g^{\nu}(x, v)=f^{\nu}\left(t^{\nu}, x, v\right), \tilde{\Psi}^{\nu}(x, z)=\Psi^{\nu}\left(t^{\nu}, x, z\right), \pi^{\nu}(x, z)=\partial_{t} \Psi^{\nu}\left(t^{\nu}, x, z\right)$, with $\left(f^{\nu}, \Psi^{\nu}\right)$ the solution of (1)-(4) determined by

$$
\left.f^{\nu}\right|_{t=0}=f_{0}^{\nu},\left.\quad \Psi^{\nu}\right|_{t=0}=\Psi_{0}^{\nu},\left.\quad \partial_{t} \Psi^{\nu}\right|_{t=0}=\Psi_{1}^{\nu},
$$

satisfy

$$
H\left(g^{\nu} \mid \mathscr{M}_{\mathrm{eq}}\right)+\frac{c^{2}}{2} \iint_{\mathbb{R}^{d} \times \mathbb{R}^{n}}\left|\nabla_{z}\left(\tilde{\Psi}^{\nu}-\Psi_{\mathrm{eq}}\right)\right|^{2} \mathrm{~d} z \mathrm{~d} x+\frac{1}{2} \iint_{\mathbb{R}^{d} \times \mathbb{R}^{n}}\left|\pi^{\nu}\right|^{2} \mathrm{~d} z \mathrm{~d} x \geq \epsilon .
$$

Conservation of the total energy (8) and conservation of the Casimir functionals (10) imply that

$$
\mathscr{J}\left(g^{\nu}, \tilde{\Psi}^{\nu}, \pi^{\nu}\right)-\mathscr{J}\left(\mathscr{M}_{\mathrm{eq}}, \Psi_{\mathrm{eq}}, \pi_{\mathrm{eq}}\right)=\mathscr{J}\left(f_{0}^{\nu}, \Psi_{0}^{\nu}, \Psi_{1}^{\nu}\right)-\mathscr{J}\left(\mathscr{M}_{\mathrm{eq}}, \Psi_{\mathrm{eq}}, \pi_{\mathrm{eq}}\right)
$$

and, of course, by mass conservation, we have

$$
\iint_{\mathbb{R}^{d} \times \mathbb{R}^{d}} g^{\nu} \mathrm{d} v \mathrm{~d} x=m
$$


Using (14), relation (19) can be rewritten as

$$
\begin{aligned}
& H\left(g^{\nu} \mid \mathscr{M}_{\mathrm{eq}}\right)+\frac{c^{2}}{2} \iint_{\mathbb{R}^{d} \times \mathbb{R}^{n}}\left|\nabla_{z}\left(\tilde{\Psi}^{\nu}-\Psi_{\mathrm{eq}}\right)\right|^{2} \mathrm{~d} z \mathrm{~d} x+\frac{1}{2} \iint_{\mathbb{R}^{d} \times \mathbb{R}^{n}}\left|\pi^{\nu}\right|^{2} \mathrm{~d} z \mathrm{~d} x \\
& +\iiint_{\mathbb{R}^{d} \times \mathbb{R}^{d} \times \mathbb{R}^{n}} \sigma_{2}\left(\tilde{\Psi}^{\nu}-\Psi_{\mathrm{eq}}\right) \sigma_{1} \star\left(g^{\nu}-\mathscr{M}_{\mathrm{eq}}\right) \mathrm{d} v \mathrm{~d} z \mathrm{~d} x \\
& =H\left(f_{0}^{\nu} \mid \mathscr{M}_{\mathrm{eq}}\right)+\frac{c^{2}}{2} \iint_{\mathbb{R}^{d} \times \mathbb{R}^{n}}\left|\nabla_{z}\left(\Psi_{0}^{\nu}-\Psi_{\mathrm{eq}}\right)\right|^{2} \mathrm{~d} z \mathrm{~d} x+\frac{1}{2} \iint_{\mathbb{R}^{d} \times \mathbb{R}^{n}}\left|\Psi_{1}^{\nu}\right|^{2} \mathrm{~d} z \mathrm{~d} x \\
& +\iiint_{\mathbb{R}^{d} \times \mathbb{R}^{d} \times \mathbb{R}^{n}} \sigma_{2}(z)\left(\Psi_{0}^{\nu}-\Psi_{\mathrm{eq}}\right)(x, z) \sigma_{1} \star\left(f_{0}^{\nu}-\mathscr{M}_{\mathrm{eq}}\right)(x, v) \mathrm{d} v \mathrm{~d} z \mathrm{~d} x .
\end{aligned}
$$

We start by observing that the right hand side tends to 0 as $\nu \rightarrow \infty$.

Lemma 3.4 We have

$$
\lim _{\nu \rightarrow \infty} \mathscr{J}\left(f_{0}^{\nu}, \Psi_{0}^{\nu}, \Psi_{1}^{\nu}\right)-\mathscr{J}\left(\mathscr{M}_{\text {eq }}, \Psi_{\text {eq }}, \pi_{\text {eq }}\right)=0 .
$$

Proof. This is a consequence of (17). The last term in (21) is nothing but $\mathscr{B}\left(\Psi_{0}^{\nu}-\right.$ $\left.\Psi_{\text {eq }}, \rho_{0}^{\nu}-\rho_{\text {eq }}\right)$, with $\rho_{0}^{\nu}(x)=\int_{\mathbb{R}^{d}} f_{0}^{\nu}(x, v) \mathrm{d} v$, and we appeal to the continuity property stated in Lemma 2.1.

It follows that

$$
\lim _{\nu \rightarrow \infty} \mathscr{J}\left(g^{\nu}, \tilde{\Psi}^{\nu}, \pi^{\nu}\right)=\mathscr{J}\left(\mathscr{M}_{\text {eq }}, \Psi_{\text {eq }}, \pi_{\text {eq }}\right)
$$

We are led to a contradiction owing to Lemma 3.3 which makes $\left(\left(g^{\nu}, \tilde{\Psi}^{\nu}, \pi^{\nu}\right)\right)_{\nu \in \mathbb{N}}$ appear as a minimizing sequence for $\mathscr{J}$ with the mass constrained to be $m$. By using Lemma 3.3- $(i i)$, we can find a suitable subsequence $\left(\nu_{k}\right)_{k \in \mathbb{N}}$ such that

$$
\lim _{k \rightarrow \infty} \iiint_{\mathbb{R}^{d} \times \mathbb{R}^{d} \times \mathbb{R}^{n}} \sigma_{2}(z)\left(\tilde{\Psi}^{\nu_{k}}-\Psi_{\text {eq }}\right)(x, z) \sigma_{1} \star\left(g^{\nu_{k}}-\mathscr{M}_{\mathrm{eq}}\right)(x, v) \mathrm{d} v \mathrm{~d} z \mathrm{~d} x=0 .
$$

Going back to (19) and (21), we are thus led to

$$
\begin{aligned}
& \lim _{k \rightarrow \infty}\left\{\mathscr{J}\left(g^{\nu_{k}}, \tilde{\Psi}^{\nu_{k}}, \pi^{\nu_{k}}\right)-\mathscr{J}\left(\mathscr{M}_{\text {eq }}, \Psi_{\text {eq }}, \pi_{\text {eq }}\right)\right\}=0 \\
& =\lim _{k \rightarrow \infty}\left\{H\left(g^{\nu_{k}} \mid \mathscr{M}_{\text {eq }}\right)+\frac{c^{2}}{2} \iint_{\mathbb{R}^{d} \times \mathbb{R}^{n}}\left|\nabla_{z}\left(\tilde{\Psi}^{\nu_{k}}-\Psi_{\text {eq }}\right)\right|^{2} \mathrm{~d} z \mathrm{~d} x+\frac{1}{2} \iint_{\mathbb{R}^{d} \times \mathbb{R}^{n}}\left|\pi^{\nu_{k}}\right|^{2} \mathrm{~d} z \mathrm{~d} x\right\} .
\end{aligned}
$$

Since the three sequences involved in the last expression are all non negative, we conclude that

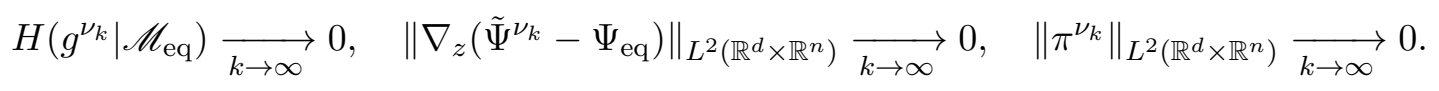

This contradicts (18).

\section{A Weak compactness in $L^{1}$ for $h(s)=s \ln (s)-s$}

The difficulty comes from the fact that $h(f)$ might change sign. We shall prove that $g^{\nu}\left|\ln \left(g^{\nu}\right)\right|$ and $\left(v^{2}+V(x)\right) g^{\nu}$ are bounded in $L^{1}\left(\mathbb{R}^{d} \times \mathbb{R}^{d}\right)$, which will allow us to apply the Dunford-Pettis theorem [16, Section 7.3.2], taking into account (H0). 
Owing to Young's inequality, we deduce from Lemma 2.1 that there exists $C>0$ such that for any $\beta>0$, we have $\mathscr{B}\left(g^{\nu}, \psi^{\nu}\right)=\iint_{\mathbb{R}^{d} \times \mathbb{R}^{d}} \Phi\left[\psi^{\nu}\right] g^{\nu} \mathrm{d} v \mathrm{~d} x \geq-\beta\left\|\nabla \psi^{\nu}\right\|_{L^{2}\left(\mathbb{R}^{d} \times \mathbb{R}^{d}\right)}-$ $\frac{C}{\beta} m^{2}$. Therefore we get

$$
\begin{aligned}
\frac{C}{\beta} m^{2}+\mathscr{J}\left(g^{\nu}, \psi^{\nu}, \pi^{\nu}\right) \geq \iint_{\mathbb{R}^{d} \times \mathbb{R}^{d}} h\left(g^{\nu}\right) \mathrm{d} v \mathrm{~d} x+\iint_{\mathbb{R}^{d} \times \mathbb{R}^{d}} g^{\nu}\left(\frac{v^{2}}{2}+V(x)\right) \mathrm{d} v \mathrm{~d} x \\
+\left(\frac{c^{2}}{2}-\beta\right)\left\|\nabla \psi^{\nu}\right\|_{L^{2}\left(\mathbb{R}^{d} \times \mathbb{R}^{d}\right)}^{2}+\frac{1}{2}\left\|\pi^{\nu}\right\|_{L^{2}\left(\mathbb{R}^{d} \times \mathbb{R}^{d}\right)}^{2},
\end{aligned}
$$

where we can pick $0<\beta<c^{2} / 2$. Since the left hand side it bounded with respect to $\nu$, it already justifies the weak compactness of $g^{\nu}, \psi^{\nu}$ and $\pi^{\nu}$ when $h$ is non negative and satisfies (H1).

For $h(s)=s \ln (s)-s$, we use the following argument: for $\Omega \geq 0$, we have

$$
\begin{aligned}
s|\ln (s)| & =s \ln (s)-2 s \ln (s)\left(\mathbf{1}_{e^{-\Omega} \leq s \leq 1}+\mathbf{1}_{e^{-\Omega}>s}\right) \\
& \leq s \ln (s)+2 \Omega s+\frac{4}{e} e^{-\Omega / 2}
\end{aligned}
$$

We use this inequality with $\Omega=2 \alpha\left(\frac{v^{2}}{2}+V(x)\right), 0<\alpha<1 / 4$. It allows us to obtain

$$
\begin{gathered}
\iint_{\mathbb{R}^{d} \times \mathbb{R}^{d}} g^{\nu}\left|\ln \left(g^{\nu}\right)\right| \mathrm{d} v \mathrm{~d} x+(1-4 \alpha) \iint_{\mathbb{R}^{d} \times \mathbb{R}^{d}} g^{\nu}\left(\frac{v^{2}}{2}+V(x)\right) \mathrm{d} v \mathrm{~d} x \\
+\left(\frac{c^{2}}{2}-\beta\right)\left\|\nabla \psi^{\nu}\right\|_{L^{2}\left(\mathbb{R}^{d} \times \mathbb{R}^{d}\right)}^{2}+\frac{1}{2}\left\|\pi^{\nu}\right\|_{L^{2}\left(\mathbb{R}^{d} \times \mathbb{R}^{d}\right)}^{2} \\
\leq \iint_{\mathbb{R}^{d} \times \mathbb{R}^{d}} h\left(g^{\nu}\right) \mathrm{d} v \mathrm{~d} x+\iint_{\mathbb{R}^{d} \times \mathbb{R}^{d}} g^{\nu}\left(\frac{v^{2}}{2}+V(x)\right) \mathrm{d} v \mathrm{~d} x \\
+\frac{c^{2}}{2}\left\|\nabla \psi^{\nu}\right\|_{L^{2}\left(\mathbb{R}^{d} \times \mathbb{R}^{d}\right)}^{2}+\frac{1}{2}\left\|\pi^{\nu}\right\|_{L^{2}\left(\mathbb{R}^{d} \times \mathbb{R}^{d}\right)}^{2}+\mathscr{B}\left(g^{\nu}, \psi^{\nu}\right) \\
+m+\frac{C}{\beta} m^{2}+\frac{4}{e} \iint_{\mathbb{R}^{d} \times \mathbb{R}^{d}} e^{-\alpha V(x)} e^{-\alpha v^{2} / 2} \mathrm{~d} v \mathrm{~d} x \\
\leq \mathscr{J}\left(g^{\nu}, \psi^{\nu}, \pi^{\nu}\right)+m+\frac{C}{\beta} m^{2}+\frac{4}{e} \iint_{\mathbb{R}^{d} \times \mathbb{R}^{d}} e^{-\alpha V(x)} e^{-\alpha v^{2} / 2} \mathrm{~d} v \mathrm{~d} x
\end{gathered}
$$

which is bounded uniformly with respect to $\nu$.

\section{References}

[1] B. Aguer, S. De Bièvre, P. Lafitte, and P. E. Parris. Classical motion in force fields with short range correlations. J. Stat. Phys., 138(4-5):780-814, 2010.

[2] R. Alonso, T. Goudon, and A. Vavasseur. Damping of particles interacting with a vibrating medium. Ann. IHP. Anal. Non-Linéaire, 2017. To appear.

[3] N Ben Abdallah and J. Dolbeault. Relative entropies for kinetic equations in bounded domains (irreversibility, stationary solutions, uniqueness). Archiv. Ration. Mech. Anal., 168(4):253-298, 2003. 
[4] H. Brézis. Analyse fonctionnelle. Théorie et applications. Masson, 1987.

[5] L. Bruneau and S. De Bièvre. A Hamiltonian model for linear friction in a homogeneous medium. Comm. Math. Phys., 229(3):511-542, 2002.

[6] M.-J Cáceres, J. A. Carrillo, and J. Dolbeault. Nonlinear stability in $L^{p}$ for a confined system of charged particles. SIAM J. Math. Anal., 34:478-494, 2002.

[7] I. Csiszar. Information-type measures of difference of probability distributions and indirect observations. Studia Sci. Math. Hungar., 2:299-318, 1967.

[8] S. De Bièvre, T. Goudon, and A. Vavasseur. Particles interacting with a vibrating medium: existence of solutions and convergence to the Vlasov-Poisson system. SIAM J. Math. Anal., 48(6):3984-4020, 2016.

[9] S. De Bièvre, P. Lafitte, and P. E. Parris. Normal transport at positive temperatures in classical Hamiltonian open systems. In Adventures in mathematical physics, volume 447 of Contemp. Math., pages 57-71. Amer. Math. Soc., Providence, RI, 2007.

[10] S. De Bièvre and P. E. Parris. Equilibration, generalized equipartition, and diffusion in dynamical Lorentz gases. J. Stat. Phys., 142(2):356-385, 2011.

[11] S. De Bièvre, P. E. Parris, and A. Silvius. Chaotic dynamics of a free particle interacting linearly with a harmonic oscillator. Phys. D, 208(1-2):96-114, 2005.

[12] J. Dolbeault, P. Markowich, and A. Unterreiter. On singular limits of mean-field equations. Arch. Rational Mech. Anal., 158(4):319-351, 2001).

[13] J. Dolbeault, C. Mouhot, and C. Schmeiser. Hypocoercivity for linear kinetic equations conserving mass. Trans. AMS, 367(6):3807-3828, 2015.

[14] J. Dolbeault, O. Sánchez, and J. Soler. Asymptotic behaviour for the VlasovPoisson system in the stellar-dynamics case. Archiv. Ration. Mech. Anal., 171:301$327,2004$.

[15] R. E. Edwards. Functional analysis. Dover Publications Inc., New York, 1995. Theory and applications, Corrected reprint of the 1965 original.

[16] T. Goudon. Intégration: Intégrale de Lebesgue et introduction à l'analyse fonctionnelle. Références Sciences. Ellipses, 2011.

[17] T. Goudon and A. Vavasseur. Mean field limit for particles interacting with a vibrating medium. Annali Univ. Ferrara, 62(2):231-273, 2016.

[18] Y. Guo. Variational method for stable polytropic galaxies. Archiv. Ration. Mech. Anal., 130:163-182, 1999.

[19] Y. Guo and G. Rein. Stable steady states in stellar dynamics. Archiv. Ration. Mech. Anal., 147:225-243, 1999.

[20] S. Kullback. A lower bound for discrimination information in terms of variation. IEEE Trans. Information Theory, 4:126-127, 1967.

[21] P. Lafitte, P. E. Parris, and S. De Bièvre. Normal transport properties in a metastable stationary state for a classical particle coupled to a non-Ohmic bath. J. Stat. Phys., 132(5):863-879, 2008. 
[22] L. Nirenberg. On elliptic partial differential equations. Ann. Scuola Norm. Sup. Pisa, Classe di Scienze, 13(2):115-162, 1959.

[23] G. Rein. Collisionless kinetic equations from astrophysics: The Vlasov-Poisson system. In Handbook of Differential Equations: Evolutionary Equations, volume 3, pages 385-476. Elsevier, 2007.

[24] E. Soret and S. De Bièvre. Stochastic acceleration in a random time-dependent potential. Stochastic Process. Appl., 125(7):2752-2785, 2015.

[25] A. Vavasseur. Some models of particles interacting with their environment. PhD thesis, University Nice Sophia Antipolis, 2016.

[26] G. Wolansky. On nonlinear stability of polytropic galaxies. Ann. Inst. H. Poincaré, 16:15-48, 1999. 